\title{
Perspectivas de la educación rural en Iberoamérica. Equidad, inclusión e innovación
}

\author{
Perspectives on rural education in Ibero-America. Equity, inclusion, innovation
}

\author{
Lucila Galván Mora \\ lucila_galvan@yahoo.com.mx \\ Red Temática de Investigación de la Educación Rural. México
}

\author{
Ana María Cadavid Rojas \\ ancadavid@udem.edu.co \\ Universidad de Medellin. Colombia
}

\section{Resumen}

Este artículo tiene el propósito principal de presentar los seis artículos que integran este Monográfico, a través de una breve reseña de cada uno de ellos. Todos son producto de investigaciones educativas realizadas por los autores y autoras, búsquedas de sentido sobre el papel histórico y político que ha jugado la escuela rural en los países de la región. Las temáticas que abordan los textos son diversas, así como las perspectivas teóricas y procedimientos metodológicos que sostienen las interpretaciones y respaldan los hallazgos. El eje articulador está dado por la intención compartida de revalorar el papel de la escuela rural en el mundo contemporáneo, y sumar esfuerzos para promover la equidad, la inclusión y la innovación educativas.

Palabras clave: educación rural; equidad; inclusión; innovación educativa.

\section{Abstract}

This article has the main purpose of presenting the six articles that make up this Monograph, through a brief review of each one of them. All are the product of educational research carried out by the authors, searches for meaning on the historical and political role that rural schools have played in the countries of the region. The topics addressed by the texts are diverse, as well as the theoretical perspectives and methodological procedures that support the interpretations and support the findings. The articulating axis is given by the shared intention to revalue the role of the rural school in the contemporary world, and join efforts to promote equity, inclusion, and educational innovation.

Keywords: rural education; equity; inclusion; educational innovation.

Recibido / Received: 10-12-2020

Aceptado / Accepted: 21-12-2020

Publicación en linea / Published online: 22-12-2020

Cómo referenciar este artículo / How to reference this article:

Galván Mora, L., \& Cadavid Rojas, A. M. (2021). Perspectivas de la educación rural en Iberoamérica. Equidad, inclusión e innovación. Tendencias Pedagógicas, 37, pp. 1-6. doi: 10.15366/tp2021.37.001 


\section{Introducción}

La educación rural es un tema de investigación que cobra cada vez mayor fuerza en los países de Iberoamérica. Podemos decir que analizar la problemática educativa en nuestros territorios responde a un interés renovado por ofrecer alternativas de mejora acordes con las características distintivas de la población rural, cercanas a sus modos de vida y atentas a sus derechos fundamentales. Los esfuerzos investigativos así emprendidos están atravesados por un afán claramente propositivo.

Las políticas educativas de algunos países de la región ignoran el valor cultural y pedagógico de la escuela rural, impulsando programas que lejos de fortalecer su sentido identitario y reconocimiento de lo propio, parecen querer borrar su especificidad con acciones derivadas de una visión urbanocéntrica de la escuela que, además de pretender homogeneizar los aprendizajes, fracturan la sinergia social en los territorios. La relevancia de la escuela rural en la construcción de la civilidad no parece ser punto de reflexión en muchos programas educativos (Cadavid, 2018).

El cierre de pequeñas escuelas rurales, ubicadas en localidades dispersas, es una política en pleno crecimiento exponencial que, con la promesa de garantizar equidad social, busca amortizar el gasto educativo concentrando a los alumnos de los centros clausurados en escuelas de mayor tamaño, con el consecuente desarraigo de su familia y lugar de origen. Esta política resulta sumamente agresiva para la población rural porque, como lo muestra la literatura, en vez de resolver la marginación, provoca mayor exclusión (Juárez, 2018).

Frente a esta realidad, pensamos un Monográfico que propicie la reflexión colectiva sobre la trascendencia histórica de la escuela rural en nuestros países. Una reflexión basada en la indagación de las condiciones en las que se lleva a cabo la labor educativa, los silenciosos -o silenciados- esfuerzos que emprenden los docentes para que la educación de niños, niñas y jóvenes sea posible, la implicación de las comunidades en la construcción -material y simbólica- de las escuelas y las oportunidades que guardan las aulas para ejercer una enseñanza innovadora.

Partimos de reconocer que la escuela rural es un espacio abierto a la comunidad y, por tanto, receptora de identidades y emociones locales (Boix, 2014). En este sentido, queremos mostrar los múltiples significados que adquiere la escuela como institución socialmente construida, desde la cotidianidad de la labor docente en los variados paisajes culturales de la región. Ofrecemos, a través de los artículos que integran este Monográfico, una mirada plural a la complejidad de la educación rural en Iberoamérica, sus claroscuros, avatares y alternativas de mejora.

\section{Un poco de historia}

La integración de un Monográfico dedicado a la educación rural en Iberoamérica fue iniciativa de algunos miembros de la Red Temática de Investigación de Educación Rural (RIER) ${ }^{1}$, interesados en difundir resultados de investigaciones sobre el campo y contribuir en los debates abiertos sobre las continuidades de la escuela rural y su horizonte de cambio posible.

Elaboramos una propuesta titulada «Una mirada a la Educación Rural en Iberoamérica. Equidad, inclusión e innovación», con la intención de explicitar el enfoque y los temas de discusión que queríamos imprimir a las colaboraciones. Tal propuesta fue enviada a la revista Tendencias Pedagógicas en marzo del año 2019, de acuerdo a su convocatoria abierta. Y en mayo de ese mismo año, recibimos el dictamen favorable para integrar el temático que ahora tienen en sus manos.

El recorrido para ver materializadas nuestras aspiraciones, resultó ser una aventura intelectual sumamente estimulante, desde la inicial integración de la propuesta, hasta la culminación de la andanza con la publicación de los artículos.

\footnotetext{
${ }^{1}$ La RIER es reconocida por el Consejo Nacional de Ciencia y Tecnología (CONACYT), organismo establecido en México. Está adscrita al Área de conocimiento «Sociedad» y al tema prioritario «Combate a la Pobreza», rubros establecidos por este mismo Consejo. La RIER tiene cobertura internacional y su sede oficial es la Universidad Iberoamericana, ubicada en Santa Fe, Ciudad de México. (http://rededucacionrural.mx/)
} 
Decidimos una coordinación conjunta no solo para compartir responsabilidades, sino para fomentar una conversación permanente sobre la situación educativa en la región y su particular expresión en los territorios rurales de nuestros países. Las dos investigadoras que rubricamos este artículo, aceptamos coordinar las actividades necesarias para llevar adelante este proyecto editorial.

De entrada, se invitó a colegas de la RIER a colaborar con artículos originales, congruentes con la estructura preestablecida para el Monográfico, que originalmente abarcaba tres grandes secciones: Políticas y currículum, Innovación y gestión pedagógicas, y Formación profesional de los docentes.

Asimismo, respetando los criterios editoriales de la revista, la invitación se abrió a otros colegas que estuvieran interesados en publicar sus trabajos sobre educación rural. Con este fin, ambas filmamos un video para exponer los propósitos del Monográfico y convocar la participación general, mismo que se difundió a través de la página de la revista. Lo filmamos en Rionegro, en el bello país de Colombia, aprovechando un encuentro académico que tuvimos en la región de Antioquia a propósito de temas sobre educación y ruralidad. ${ }^{2}$

Vimos con satisfacción la respuesta favorable a las convocatorias emitidas. Llegaron textos de distintas latitudes, que abordaban temáticas tan diversas como heterogéneo y complejo es el campo de la educación rural en Iberoamérica.

Después de los dictámenes, los tiempos para la corrección de los artículos y los plazos para la entrega de versiones finales, se seleccionaron 6 artículos que ubican al lector en escenarios rurales de México, Argentina, Colombia, El Ecuador, España y Cuba. Invitamos, a través de su lectura, a realizar un recorrido por las incontables aristas que contiene le tema. A mirar, a través de quienes escribieron los textos, los múltiples desafíos y oportunidades de la educación rural en estos territorios.

\section{Estructura}

El propósito principal de nuestro texto es presentar los artículos que integran el Monográfico, a través de una breve reseña de cada uno de ellos. Todos son producto de investigaciones educativas realizadas por los autores y autoras, búsquedas de sentido sobre el papel histórico y político que ha jugado la escuela rural en los países de la región. Las temáticas que abordan los textos son diversas, así como las perspectivas teóricas y procedimientos metodológicos que sostienen las interpretaciones y respaldan los hallazgos. El eje articulador está dado por la intención compartida de revalorar el papel de la escuela rural en el mundo contemporáneo, y sumar esfuerzos para promover la equidad, la inclusión y la innovación educativas.

«Programas educativos dirigidos a poblaciones de los territorios rurales. Experiencias en Argentina y México», de Diego Juárez Bolaños, encabeza la serie. Se trata de un texto ameno e interesante, que examina intenciones y alcances de dos programas de educación rural. Uno de ellos implementado en la Provincia de Buenos Aires, Argentina; el otro, en el Estado de Campeche, en México.

El contraste entre los dos programas no se reduce a la particularidad de los contextos donde operan, el autor advierte también las distintas filiaciones institucionales y políticas que éstos representan. El programa de Argentina, tributario de una Pedagogía de alternancia, establece para los alumnos que habitan territorios con alta dispersión, estancias acotadas en las escuelas y periodos de actividades escolares en casa, apoyados por tareas previamente planeadas y visitas sistemáticas de los docentes. El programa de México, vinculado a una política de concentración de servicios educativos, ofrece rutas de trasporte para trasladar alumnos de localidades dispersas a centros educativos ubicados en zonas urbanizadas, con el consecuente cierre de las pequeñas escuelas rurales.

Las implicaciones de estos programas para las familias rurales también son contrastantes. En el primer caso, el programa se distingue por su apoyo a la producción total de la región y es cogestionado por el Estado y las familias. Las escuelas promueven procesos de organización comunitaria y desarrollo local, se ocupan de la extensión agraria y la capacitación tecnológica para los productores. En el caso mexicano, las familias participan con recelo. Algunas, incluso envían a sus hijos a escuelas más

2 Nos referimos al Cuarto Coloquio Iberoamericano de Educación Rural, que se llevó a cabo en el marco del Congreso Internacional Epistemologías del Sur y Ruralidades Latinoamericanas, del 9 al 11 de octubre de 2019, en Rionegro-Antioquia, Colombia. 
cercanas que las indicadas por el programa, solventando el traslado con sus propios recursos; otras, enfrentan con preocupación la inadaptación que sufren los alumnos, sobre todo los mas pequeños, al estar lejos de su familia y su comunidad. Las consecuencias de este desarraigo provocado no se han sopesado aún con suficiente mesura.

$\mathrm{El}$ autor ofrece un análisis al tratamiento de las necesidades educativas de la población rural, que implica todo programa educativo. En algunos casos, éstos pueden fomentar el arraigo a la comunidad y la sinergia del territorio; en otros, el desprendimiento de la localidad y el despoblamiento. Con talante crítico, hace un cuestionamiento severo a la política de cierre de escuelas rurales, que pasa por alto el derecho de la población a recibir educación en sus propios lugares.

«Las guías de aprendizaje: el currículo que se define para la escuela primaria rural desde el Modelo Escuela Nueva en Colombia», de Ana María Cadavid Rojas, ofrece un cuidadoso análisis de las disposiciones curriculares implicadas en los materiales de estudio que se usan de manera predominante en las escuelas de contextos rurales. Desde una perspectiva crítica del currículo, la autora define a las guías de aprendizaje vinculadas al modelo Escuela Nueva, como dispositivos que configuran prácticas de enseñanza, trasmiten ideologías y definen relaciones que configuran una determinada estructura escolar, orientada a la estandarización de la enseñanza y la unificación del currículo en el territorio nacional.

El estudio cualitativo sobre los usos y desusos de las guías de aprendizaje, que ella llevó a cabo en primarias colombianas, muestra algunas incidencias que remiten a la invisibilidad de la escuela rural, como institución con características propias y orientaciones curriculares específicas para responder a los contextos locales, a la diversidad de ruralidades que se encuentran en el país. A través de las guías de aprendizaje, como centro del modelo educativo, se promueve la homogeneidad del currículo bajo la idea equivocada de que así se fomenta la equidad educativa y social en los sectores de población rural más desfavorecidos.

El artículo pone en la mesa de discusión el componente curricular de la llamada Escuela Nueva de Colombia. Las orientaciones didácticas preestablecidas, los tiempos marcados por la uniformidad, la graduación lineal de los contenidos y los aprendizajes esperados definidos sin contemplar la evidente diversidad de los alumnos, constituyen puntos de inflexión de esta propuesta educativa que requiere ser revisada y replanteada.

El mensaje que trasmite la lectura de este texto es una búsqueda de congruencia entre los afanes institucionales por llevar educación a la población del campo y las diversas formas de ser y habitar los mundos rurales. En el fondo, encontramos una profunda preocupación por la formación de ciudadanos con raíces locales y pensamiento universal, en un país que día a día construye la paz.

«El acoso escolar en estudiantes con necesidades educativas especiales en una zona rural de El Ecuador», es un artículo de autoría colectiva. En éste, Rosa $\mathrm{M}^{a}$ Espada (España), Sergio Sánchez (España) y Rosa A. Sánchez (El Ecuador), abordan con rigurosidad un problema actual que se presenta en los centros educativos: el acoso escolar o bullying. Se basan en un estudio realizado en escuelas rurales de la provincia de Machala, en El Ecuador; a través de una muestra de ciento quince estudiantes de séptimo grado, con 10.9 años promedio. Algunos de estos estudiantes, que presentaban necesidades educativas especiales (NEE) con o sin discapacidad, fueron el centro de la discusión que las autoras y el autor emprenden a propósito de la inclusión educativa en entornos rurales.

$\mathrm{El}$ acoso se define como una forma de violencia entre pares, manifestada como agresión física o psicológica, como humillaciones, rechazo o marginación del grupo. Constituye, ahora mismo, un tema de atención prioritaria en la mayoría de los sistemas educativos de la región. No obstante, quienes escriben el artículo afirman que los programas de atención que intentan solucionar o prevenir el acoso escolar, no toman en cuenta la variable de discapacidad que presentan algunos alumnos.

Esta situación se agudiza en contextos rurales, en los cuales la relación de dominio-sometimiento que implica el acoso parece un fenómeno normalizado, que se oculta con el hermetismo de las familias, la carencia de recursos adecuados o la dificultad de los profesores para tratar adecuadamente el problema. La escuela rural presenta en este tema una gran brecha de atención, que la convierte en un espacio más de exclusión social de la población más vulnerable, como sería los niños y niñas con NEE. Así, estos infantes constituyen los sujetos diana como víctimas del acoso y la marginación.

El artículo muestra datos duros sobre las formas de acoso que sufren estos colectivos infantiles en las aulas. Y lanza una llamada de atención a las autoridades educativas para revertir este tipo de situaciones que lesionan la integridad de los alumnos, su derecho a ser respetados y ser incluidos en los complejos mundos escolares. 
En la perspectiva de indagar por la formación de los docentes para la escuela rural, se encuentra el artículo «Análisis de la formación inicial en escuela rural en los Grados de Magisterio: valoraciones y percepciones del alumnado como agentes implicados», de Silvia Anzano Oto, Sandra Vázquez Toledo y Marta Liesa Orús de la Universidad de Zaragoza. La investigación, con una apuesta de indagación cuantitativa, analiza desde el lugar de los docentes de la comunidad aragonesa de España, si la formación universitaria que recibieron durante sus cuatro años en los Grados de Magisterio fue pertinente y solvente para trabajar en la escuela rural.

Resalta el encuadre referido a la normativa sobre la formación para la escuela rural, así como los análisis que presentan los autores sobre las discusiones que, en el marco de distintas investigaciones, se han dado sobre la necesidad de intencionar la formación de los docentes que enseñarán en contextos rurales, de tal manera que conozcan con profundidad aspectos vinculados a la dimensión territorial, educativa y didáctica de la labor docente.

El estudio concluye que es necesaria una formación específica, que no se limite a algunos cursos desarticulado sin contemplar de manera sistemática espacios de práctica que permitan conocer las escuelas rurales para la construcción de propuestas educativas pertinentes, en diálogo con los contextos en los que se encuentran. Así mismo, los autores señalan, a partir de lo expresado por los alumnos, la necesidad que existe de repensar los programas de formación universitaria y sus propuestas para la escuela rural, de manera que ésta no se limite a asuntos solamente teóricos sino también abarque experiencias de práctica y construcción didáctica.

Por su parte el artículo «Análisis de dificultades en la enseñanza y aprendizaje del español y las matemáticas en escuelas primarias multigrado de Veracruz-México», elaborado por Amanda Cano Ruiz desde una aproximación cuantitativa, indaga sobre la educación multigrado en México desde las consideraciones de los docentes que trabajan con dicha metodología en escuelas primarias.

Resulta interesante que, desde la aplicación de un cuestionario a docentes multigrado, ellos señalen o mejor dicho reiteren los problemas que han acompañado la historia de la educación rural, como son: las precarias condiciones de infraestructura en las que se lleva a cabo la labor docente y los pocos materiales con los que cuentan para la enseñanza.

De manera muy clara, el artículo se acerca a las reflexiones y análisis que realizan los docentes con respecto al logro de los aprendizajes en las asignaturas de español y matemáticas. Para ellos, las características sociales, culturales y familiares de los alumnos, son condiciones que convergen en las dificultades que presentan para la comprensión textual y la resolución de problemas matemáticos.

En esta dirección, el artículo visibiliza la necesidad de fortalecer en los docentes la formación didáctica para la enseñanza de las matemáticas y el español, así como para enfrentar los retos del trabajo con grupos multigrado. Este asunto es clave porque sitúa también la importancia de repensar para transformar la formación inicial de docentes para los contextos rurales, formación que en la mayoría de los casos ha estado invisible en las políticas educativas o se ha restringido a asuntos superficiales, desconociendo la potencia didáctica y pedagógica del trabajo que los docentes realizan en las escuelas rurales multigrado. Entre las conclusiones que presenta la investigación, resalta la importancia de centrar la mirada en la labor docente que realizan los maestros multigrado, para conocer las elaboraciones didácticas que se construyen en las aulas, aportes y experiencias que pueden ser referentes a considerar en programas específicos de formación continua.

Para finalizar las contribuciones sobre los debates, posibilidades y retos con respecto a la educación rural, encontramos el artículo de María Fidelia Díaz Reyes y Estrella Margarita Lardoeyt Llauradó de Cuba, titulado «¿Cómo cultivar la originalidad en la escuela primaria rural?». La investigación aborda el proceso de enseñanza-aprendizaje de la lengua materna, en el cual el maestro pone en juego su reflexión didáctica y diseña procesos de intervención con el propósito de propiciar el desarrollo de la comprensión lectora y la originalidad en los estudiantes de un aula multigrado.

El trabajo se realiza en contextos rurales con estudiantes de quinto grado desde la apuesta por mejorar los procesos de aprendizaje a partir de un diagnóstico de las debilidades y potencialidades de los alumnos. Resulta muy interesante la noción de originalidad con la que se realiza la investigación, porque además de considerar el trabajo individual, promueve la construcción de textos de manera colectiva por parte de los estudiantes. El artículo es un aporte al campo de la enseñanza de la lectura y la escritura en educación rural, tema de gran importancia para continuar indagando. 


\section{A manera de cierre}

Desde este foro, hacemos una invitación a leer los artículos expuestos en este Monográfico, que representan 6 miradas acuciosas a los retos y oportunidades de la escuela rural en el vasto territorio iberoamericano. Miradas críticas, propositivas y nutridas con el anhelo de una mejor educación para nuestros los pueblos rurales, congruente con sus historias y sus derechos fundamentales.

En sus páginas encontrarán discusiones centrales relativas a la equidad e inclusión educativa que requiere la escuela rural, en los variados contextos culturales de la región; así como distintos acercamientos a la potencialidad pedagógica de la escuela para responder a las necesidades y expectativas de sectores sociales que, en muchos casos, han sido olvidados por las políticas públicas.

El recorrido representa un viaje por la diversidad del territorio y sus escuelas. Estamos convencidas que será de interés para quienes pugnan por una educación diversificada, adecuada a las características particulares de cada núcleo de población y cada ruralidad, positiva a la pluralidad de expectativas y emociones que se tejen socialmente en torno a escolaridad. Interesante sobre todo para quienes investigan y trabajan por escuelas rurales que conserven su identidad, en diálogo con los contextos y con las experiencias vitales de los docentes y estudiantes.

\section{Agradecimientos}

Manifestamos nuestra gratitud a todos y todas las colegas que respondieron con entusiasmo a la convocatoria de este Monográfico y apuestan por una educación rural realmente inclusiva y equitativa. Agradecemos la colaboración de Lydia Espinosa Gerónimo, colega de la RIER, para la traducción al inglés del resumen de este artículo.

\section{Referencias}

Boix, R. (2014). La escuela rural en la dimensión territorial. Innovación Educativa, 24, 89-97.

Cadavid, A. M (2018). Educación rural en Colombia, algunas miradas y reflexiones: Retos para la reconstrucción del país. Seminario Interno de investigación del Instituto de Investigaciones para el Desarrollo de la Educación, Universidad Iberoamericana, México.

Juárez, D. (2018). Políticas de cierre de escuelas rurales en América Latina. III Coloquio Iberoamericano de Educación Rural, Cuzco, Perú. https://inide.ibero.mx/assets_front/assets/libros/2020/librocierreescuelas-digital.pdf 